\title{
Expression of MET and SOX2 genes in non-small cell lung carcinoma with EGFR mutation
}

\author{
YI-RAN CAI $^{1}$, HAI-QING ZHANG ${ }^{1}$, YANG QU ${ }^{1}$, JING MU $^{1}$, DAN ZHAO ${ }^{1}$, \\ LI-JUAN ZHOU ${ }^{1}$, HONG YAN ${ }^{1}$, JIAN-WEI YE ${ }^{2}$ and YAN LIU ${ }^{2}$ \\ ${ }^{1}$ Department of Pathology, Beijing Chest Hospital, Capital Medical University; ${ }^{2}$ Division of \\ Molecular Medicine, Wu Jieping Medical Foundation, Beijing 100144, P.R. China
}

Received April 13, 2011; Accepted May 27, 2011

DOI: 10.3892/or.2011.1349

\begin{abstract}
Non-small cell lung carcinoma (NSCLC) is a leading cause of cancer-related deaths. Aberrance of the two oncogenes MET and SOX2 are frequently encountered in NSCLC. Exons 18 through 21 of the EGFR gene were screened and MET and SOX2 immunostaining was conducted to analyze the immunohistological staining of MET and SOX2 and the EGFR mutation status. One hundred and fifty tissue samples were examined including 57 squamous cell carcinomas (SCCs), 80 adenocarcinomas (ADCs), 9 adenosquamous carcinomas (ADSCs) and 4 large cell carcinomas (LCCs). The 32 NSCLCs harboring an EGFR mutation included 28 ADCs, 3 SCCs and 1 ADSC. A higher level of SOX2 expression appeared in NSCLCs without the EGFR mutation compared to those with EGFR mutation $\left(\chi^{2}=9.02, \mathrm{P}=0.0027\right)$. Of the $28 \mathrm{ADCs}$, $24(85.7 \%)$ with an EGFR mutation showed low level of SOX2 expression. ADCs with deletion in exon 19 overexpressed MET and showed low levels of SOX2. SOX2 expression was inversely correlated to the expression of MET in NSCLC and mainly present in non-mutated NSCLC $(r=-0.42, \mathrm{P}<0.0001)$. There was a tendency for SOX 2 to be expressed in SCCs and particularly in the part of SCC among ADSCs, whereas MET was mainly expressed in the part of ADC among ADSCs and ADCs. High level of MET and SOX2 expression were respectively demonstrated in ADCs and SCCs; MET activation was accompanied with exon 19 deletion in ADCs. EGFR and MET coordinate to drive tumorigenesis. Detection of the activation of MET and EGFR may be used for targeted drug therapy.
\end{abstract}

\section{Introduction}

Non-small cell lung carcinomas (NSCLCs) represent $80 \%$ of lung cancers, further classified into adenocarcinoma (ADC)

Correspondence to: Dr Yi-Ran Cai, Department of Pathology, Beijing Chest Hospital, Capital Medical University, 97 Ma Chang, Tongzhou District, Beijing 101149, P.R. China

E-mail: cyr7172@gmail.com

Key words: non-small cell lung carcinoma, MET gene, SOX2 gene, EGFR mutation, immunohistochemistry including bronchioloalveolar carcinoma (BAC) and squamous cell carcinoma (SCC) (1). Adenocarcinomas (ADC), squamous cell carcinomas (SCC) and adenosquamous carcinomas (ADSC) are three main histological subtypes of NSCLCs. Receptor tyrosine kinases (RTKs) regulate many key processes in mammalian development, cell function and tissue homeostasis. Alterations at the level of the receptor and its ligand lead to the activation of a number of signaling pathways, each of which may contribute to cancer progression. Deregulations of RTKs by mutation, gene rearrangement, gene amplification and overexpression of both receptor and ligand have been implicated as causative factors in the development and progression of numerous human cancers (2-4). The tyrosine-kinase epidermal growth factor receptor (EGFR) pathway has been shown to play an essential role in the pathogenesis of NSCLC, leading to the development of targeted therapeutic agents using small molecules of EGFR tyrosine kinase inhibitors (TKIs) such as gefitinib or erlotinib (5-7). Recent clinical evidence of EGFR-TKIs in refractory and advanced NSCLCs have raised hopes that targets of other deregulated growth factor signaling pathways, such as the hepatocyte growth factor (HGF)/MET pathway may offer alternative therapeutic treatments for NSCLC. In addition, a recent study showed that MET amplification leads to gefinitib secondary resistance and could be an explanation for this resistance in some patients (8). MET gene is the prototypic member of a subfamily of RTKs. The MET RTK family is structurally distinct from other RTK families and is the only known high-affinity receptor for HGF, also known as scatter factor (SF) $(9,10)$. In addition to proliferative and anti-apoptotic activities that are common to many growth factors, MET elicits unique mitogenic and morphogenic effects by stimulating cell-cell detachment, migration, invasiveness, tubule formation and branching $(11,12)$. These activities of the MET signaling pathway have provided examples of the mechanisms by which this pathway is involved in tumor development and progression. MET is usually considered as an oncogene (13) and appears to be implicated especially in ADCs. Cigarette smoking induces overexpression of HGF in type II alveolar pneumocytes and lung cancer cells (14). Overexpression of HGF in lung cancer cells induces alveolar differentiation/proliferation and MET activation and may play special roles in well-differentiated lung ADCs (15-17). 
The transcription factor SOX2, a member of the SRY-high mobility box transcription factor family, is expressed in epithelial cells of the foregut, including pharynx, esophagus, trachea, bronchi and bronchioli, but is excluded from the peripheral and alveolar regions of the lung (18). SOX2 is also expressed in the developing respiratory epithelium and is restricted to conducting airways of the mature lung (19). SOX2 is induced in the bronchiolar epithelium during repair after toxicant-induced injury (20). Overexpression of SOX2 in lung epithelium during early development disrupted branching morphogenesis, causing cystic lungs and neonatal death (19). It has been suggested that SOX2, which belongs to group B of the SOX family plays critical roles in cell fate determination, differentiation and proliferation $(21,22)$. A recent study identified SOX2 amplification on band 3q26.3 and intense SOX2 immunostaining in lung SCC, indicating the potentially active transcriptional regulation by SOX2. SOX2-overexpressing lung epithelial cells and embryonic stem cells (ESCs) reveal that SOX2 contributes to activation of ESC-like phenotypes providing clues pertaining to the deregulated genes involved in the malignant phenotype (23). Few data are available about MET and SOX2 expression and the EGFR mutation status in NSCLCs. The aim of our study was to simultaneously analyze MET, SOX2 and EGFR status in an NSCLC cohort to better understand the potential role of the MET and SOX2 signaling pathway and to demonstrate MET and SOX2 gene expression to its clinicopathological features.

\section{Materials and methods}

Tissue specimens. We analyzed 150 primary Chinese lung tumor specimens from patients whose surgery was performed in the Beijing Chest Hospital from 2007-2009. Resected tumors were formalin-fixed and paraffin-embedded until DNA was extracted. Corresponding non-malignant peripheral lung tissue was also available. All specimens were reviewed by two reference pathologists (H.Q.Z. and Y.R.C.) to confirm diagnosis and the predominance $(>70 \%)$ of cancer tissue in the tumor specimens. Bronchioloalveolar carcinoma (BAC) was defined as previously described (24). Both observers were blinded to patient outcomes. Clinicopathological characteristics including age, gender, histologic subtype and feature of tumor were available.

DNA extraction. Genomic DNA was derived from tumors of formalin-fixed and paraffin-embedded blocks. Tissues (50-100 mg) were scraped off the block, deparaffinized twice in xylene, rinsed twice with absolute ethanol and washed with pure water. Tissues were suspended in $500 \mu 1$ of GA containing 100-200 $\mu \mathrm{g}$ of proteinase K (Promega, Madison, WI, USA) and incubated overnight at $55^{\circ} \mathrm{C}$. Finally, DNA was purified through columns (DNeasy tissue kit, Tiangen, Beijing, China) following the directions of the manufacturer. The lysis mixture was centrifuged for 1 min to remove undigested tissue; the eluent (DNA) was combined with $200 \mu 1$ of GB buffer, vortexed and incubated at $70^{\circ} \mathrm{C}$ for $10 \mathrm{~min}$. After the addition of $210 \mu \mathrm{l}$ of $100 \%$ ethyl alcohol, the specimen was vortexed, added to a spin column and centrifuged at $9000 \mathrm{rpm}$ for $1 \mathrm{~min}$. The filtrate was discarded, $500 \mu 1$ of GD buffer were added, and the column was centrifuged at $8000 \mathrm{rpm}$ for $1 \mathrm{~min}$. The filtrate was discarded, and the preceding step was repeated twice with column spin rinsed with PW buffer. The DNA was then suspended in $100 \mu \mathrm{l}$ of TE [10 mM Tris (pH 8), $1 \mathrm{mM}$ EDTA (pH 8)]. The DNA concentration was measured by UV absorbance at $260 \mathrm{~nm}$ and then stored at $4^{\circ} \mathrm{C}$ until further use.

Polymerase chain reaction amplification and direct sequencing for EGFR mutation. We used $100 \mathrm{ng}$ genomic DNA of tumor cells as template in PCR reactions to amplify DNA fragments corresponding to exons 18-21 of EGFR. The primers were designed as follows: exon 18 forward 5'-CAACC AAGCTCTCTTGAGGATC-3', reverse 5'-CCCAGCCCAG AGGCCTGT-3'; exon 19 forward 5'-GCAGCATGTGG CA CCATCTC-3', reverse 5'-AGAGCCATGGACCCCCACAC-3'; exon 20 forward 5'-CACACTGACGTGCCTCTCC-3', reverse 5'-AGCAGGTACTGGGAGCCAAT-3'; exon 21 forward 5'-TCTGTCCCTCACAGCAGGGTCT-3', reverse 5'-GCTG GCTGACCTAAAGCCACC-3'. Template DNA was performed in $50 \mu \mathrm{l}$ volumes containing 0.75 units of Hot Start Taq DNA polymerase (Fermentas Inc., Ontario, Canada), $5 \mu \mathrm{l}$ of PCR buffer, $0.8 \mu \mathrm{M}$ dNTP, $0.5 \mu \mathrm{M}$ of each primer, and different concentrations of $\mathrm{MgCl}_{2}$, depending on the polymorphic marker. The PCR analyses were performed by 40 cycles consisting of a denaturation step at $94^{\circ} \mathrm{C}$ for $45 \mathrm{sec}$, a primer annealing step at $56^{\circ} \mathrm{C}$ for $30 \mathrm{sec}$ and an elongation step at $72^{\circ} \mathrm{C}$ for $30 \mathrm{sec}$. The final step at $72^{\circ} \mathrm{C}$ was extended for $10 \mathrm{~min}$. Sequencing of each sample was performed in duplicate with an ABI PRISM 310 analyzer (Applied Biosystems, Foster City, CA, USA). PCR products were sequenced in both sense and antisense directions. EGFR mutations detected in the initial round of sequencing were confirmed by subsequent rounds of independent PCR and sequencing reactions. Only specimens in which a mutation was identified in both rounds were recorded as mutation-positive. The nucleic acid used for mutations was based on NM_005228.3.

Immunohistochemistry. Representative formalin-fixed paraffin-embedded tissue blocks from the cohort of these 150 cases of primary NSCLC were available and found suitable for immunohistochemical study. Sections $(4 \mu \mathrm{m})$ prepared from paraffin blocks were deparaffinized using xylene and rehydrated through an ethanol series to water. Antigen retrieval was performed using a decloaking chamber at 15 psi for $15 \mathrm{~min}$ in sodium citrate buffer ( $\mathrm{pH}$ 6.0). Endogenous peroxidase enzyme activity was blocked by $3 \% \mathrm{H}_{2} \mathrm{O}_{2}$ in methanol for $30 \mathrm{~min}$ at room temperature. Slides were immunostained with rabbit polyclonal anti-Met antibody (sc-10; Santa Cruz Biotechnology, Inc., CA), and monoclonal anti-SOX2 (3579S; Cell Signaling Technology, Inc., USA) using a modification of the avidin-biotin-peroxidase method. Incubation with the primary antibody was performed overnight at $4^{\circ} \mathrm{C}$ and at a 1:200 dilution. The PowerVision system was used for immunostaining detection (PV6118, Novocastra, UK), following the manual. Detection was accomplished using the DAB reagent (ImmunoCruz Staining System, Santa Cruz Biotechnology). Slides were then counterstained with hematoxylin, and the area within the diagnostic area was scored by three independent observers on the basis of criteria that 1000 cells of each case were observed to count the percentage of positive cells in the sections. Nuclear staining was considered positive for 
A

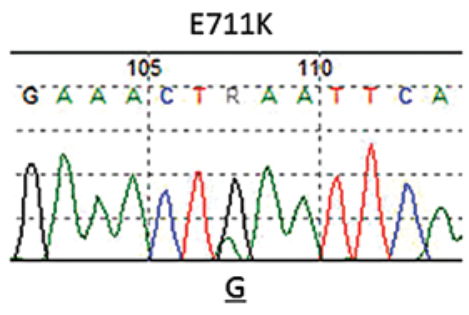

A

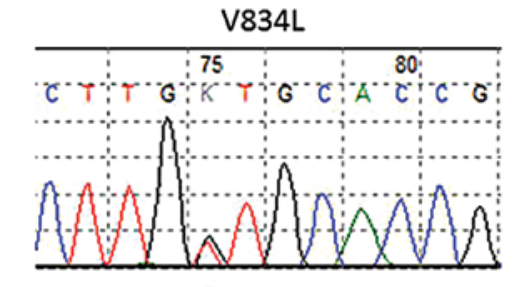

$\underline{G}$
B

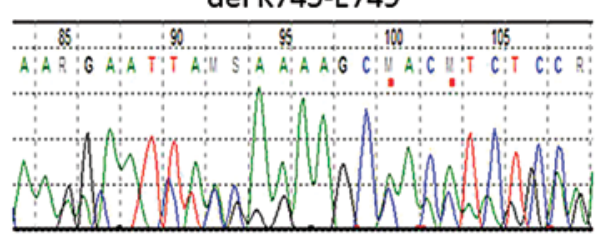

A A G GA ATTAAG A GAGCA ACATCTCCG A A A ACATCTCCGAAAG C CAACAAGG A A

2235_2249deIGGAATTAAGAGAAGC
D

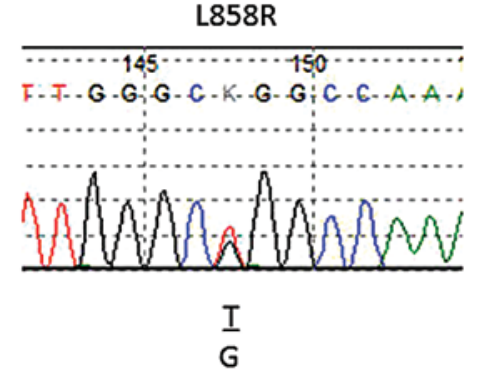

Figure 1. Mutations in the EGFR gene in NSCLCs. (A-D) Nucleotide sequence of the EGFR gene in tumor specimens with heterozygous mutation within the tyrosine kinase domain (double peaks). The double peaks represent two nucleotides at the site of heterozygous mutations. Tracings in sense directions are shown to demonstrate the mutation and deletion in the EGFR mutation hotspots; the wild-type nucleotide sequence is shown in capital letters and the mutant sequence is in lowercase letters. (B) The 5' breakpoint of del K745-E749 mutation occurs in exon 19 and make tumor sensitive to target therapy of gefitinib. (A), (C) and (D) Heterozygous missense mutations (arrows) in exons 18, 20 and 21 resulting in amino acid substitutions within the tyrosine kinase domain.

SOX2, whereas cytoplasmic staining was considered positive for MET. Immunoreactivity in the carcinoma samples was semi-quantitatively evaluated for SOX2 and MET staining by percentage of positive cells and staining: $0,1,2$ and 3 indicate that $<25 \%, 25-50 \% 51-75 \%$ and $\geq 76 \%$ tumor cells were reactive, respectively; staining criteria: 0 , tumor cells were not stained; 1, tumor cells with light-yellow stain; 2, with yellow stain; 3, with brown stain. The final score for statistical analysis was the product of multiplication both in positive cells and staining. The scores of was considered into different categories: 0 was negative (0); scores 1 was $1+; 2-6$ was $2+$; $\geq 7$ was $3+$. Furthermore, 0 and 1+ was classified as low-expressed, 2+ and $3+$ were overexpressed.

Statistical analysis. Correlations of SOX2 and MET expression with clinicopathological factors were analyzed by the crosstab $\chi^{2}$ test or the Fisher's exact test. The correlation between expression of MET and SOX2 were assayed by $r$ value for correlation. The non-parametric ANOVA test was used to analyze the nonnormal distribution data as staining scores of SOX2 and MET. All statistical tests were two-sided, significant level $\alpha=0.05$. All statistical analyses were performed using SAS version 9.2 (SAS Institute Inc., Cary, NC, USA) for Windows.

\section{Results}

Patient characteristics and histopathological features. The patients of our 150 NSCLCs were primarily men $(65.3 \%$, $\mathrm{n}=98$ ). The median age was 58 years (range, 27-77 years). Patient diagnoses included 57 with SCC (38\%), 80 with ADC (40\%) including 10 BACs, 6 mucinous carcinomas and 4 papillary adenocarcinomas (PADC), 9 with $\operatorname{ADSC}(6 \%)$ in addition to 4 large cell carcinomas (LCCs). Seventy-six patients $(50.7 \%)$ were presented with lymph node metastases. Thirteen tumors (8.7\%) were well-differentiated only in ADCs. Of the 57 cases of the SCC subgroup, tumor dimension in 28 cases (49.1\%) was $<5 \mathrm{~cm}$ and all 57 cases were moderately or poorly differentiated. Additionally, 26 cases (45.6\%) with local lymph node invasion were detected. Of the 80 cases of the ADC subgroup, 68 cases $(85 \%)$ had a tumor dimension $<5 \mathrm{~cm}, 67$ cases $(83.8 \%)$ were moderately or poorly differentiated and 45 cases (56.3\%) were female. ADSCs were histologically composed of adenocarcinoma and squamous cell carcinoma patterns. As a special entity in histological types, ADSC was used as a liaison to assess the relationship between ADC and SCC. In this subgroup, there were 6 cases with their tumor dimensions $<5 \mathrm{~cm}$, and 4 cases showed lymph node metastases. All these 9 ADSCs were moderately or poorly differentiated. Four large cell carcinomas were $<5 \mathrm{~cm} ; 3$ of them were involved in lymph node invasion.

EGFR mutation in lung NSCLC. In this study, screening for mutations of the kinase domain (exons 18 through 21) of EGFR by direct sequencing of DNA isolated from 150 lung-tumorresected specimens identified a $\mathrm{T} \rightarrow \mathrm{G}$ mutation at nucleotide 2573 of exon 21, resulting in L858R (Fig. 1). Mutations were detected in $32(21.3 \%)$ NSCLC specimens and are listed in Table I. Seventeen (11.3\%) patients harbored deletions in the region of K745-S753 of exon 19, 8 cases (5.3\%) of the L858R in exon $21,3(2 \%)$ of the E711K and G724S in exon 18 and $3(2 \%)$ of L861Q in exon 21. Two patients carried composite mutations (Table I). Twenty-eight (35\%) of ADCs, $3(5.3 \%)$ of SCCs and $1(11.1 \%)$ of ADSCs presented EGFR mutations but not the LCCs. Among these mutated cases, 23 were tubular 
Table I. Different mutations of EGFR in exon 18 through exon 21 in NSCLCs.

\begin{tabular}{|c|c|c|c|c|}
\hline $\begin{array}{l}\text { Case } \\
\text { no. }\end{array}$ & $\begin{array}{l}\text { Histological } \\
\text { types }\end{array}$ & $\begin{array}{l}\text { EGFR } \\
\text { mutation }\end{array}$ & $\begin{array}{l}\text { SOX2 } \\
\text { score }\end{array}$ & $\begin{array}{l}\text { MET } \\
\text { score }\end{array}$ \\
\hline 2 & $\mathrm{ADC}$ & L858R & 0 & 6 \\
\hline 6 & $\mathrm{SCC}$ & $\mathrm{E} 711 \mathrm{~K}$ & 6 & 2 \\
\hline 10 & $\mathrm{BAC}$ & G724S, L861Q & 0 & 1 \\
\hline 23 & $\mathrm{BAC}$ & del E746-A750 & 0 & 3 \\
\hline 27 & $\mathrm{ADC}$ & del E746-A750 & 1 & 2 \\
\hline 31 & $\mathrm{SCC}$ & L861Q & 0 & 3 \\
\hline 34 & BAC & del E746-A750 & 0 & 2 \\
\hline 39 & $\mathrm{ADC}$ & del K745-E749 & 0 & 4 \\
\hline 56 & $\mathrm{SCC}$ & L858R & 0 & 6 \\
\hline 57 & ADSC & del K745-E749 & 6 & 1 \\
\hline 59 & $\mathrm{ADC}^{\mathrm{a}}$ & del K745-E749 & 0 & 3 \\
\hline 60 & $\mathrm{ADC}$ & del K745-E749 & 1 & 3 \\
\hline 71 & $\mathrm{ADC}$ & del E746-A750 & 0 & 3 \\
\hline 73 & $\mathrm{ADC}$ & L858R & 0 & 6 \\
\hline 86 & $\mathrm{ADC}$ & del E746-A750 & 1 & 4 \\
\hline 104 & $\mathrm{ADC}$ & del K745-E749 & 0 & 2 \\
\hline 105 & $\mathrm{ADC}$ & del K745-E749 & 0 & 2 \\
\hline 115 & $\mathrm{ADC}$ & $\mathrm{D} 807 \mathrm{~N}$ & 0 & 4 \\
\hline 119 & $\mathrm{ADC}$ & L858R & 2 & 3 \\
\hline 121 & $\mathrm{ADC}$ & L858R & 0 & 6 \\
\hline 122 & $\mathrm{ADC}^{\mathrm{b}}$ & del L747-S753 & 0 & 3 \\
\hline 123 & $\mathrm{ADC}$ & del E746-A750 & 0 & 4 \\
\hline 126 & $\mathrm{ADC}$ & R776C, L858R & 4 & 6 \\
\hline 128 & $\mathrm{ADC}$ & L858R & 2 & 2 \\
\hline 129 & $\mathrm{ADC}$ & S768L & 2 & 2 \\
\hline 130 & $\mathrm{ADC}$ & del L747-E749 & 0 & 6 \\
\hline 132 & $\mathrm{ADC}$ & del E746-A750 & 0 & 3 \\
\hline 133 & $\mathrm{ADC}$ & L858R & 0 & 6 \\
\hline 142 & $\mathrm{ADC}$ & L861Q & 0 & 6 \\
\hline 144 & $\mathrm{ADC}$ & del K745-E749 & 0 & 3 \\
\hline 145 & $\mathrm{ADC}$ & $\begin{array}{l}\text { c. } 2300-2301 \\
\text { insCAGCGTGGA }\end{array}$ & 1 & 2 \\
\hline 148 & $\mathrm{ADC}$ & del K745-E749 & 0 & 4 \\
\hline
\end{tabular}

${ }^{a}$ Mucinous ADC; ${ }^{b}$ papillary ADC. BAC, adenocarcinoma with any element of bronchoalveolar carcinoma (BAC); EGFR, epidermal growth factor receptor gene; ADC, adenocarcinoma; SCC, squamous cell carcinoma.

adenocarcinoma, 3 BACs, 1 ADSC, 1 mucinous adenocarcinoma, 1 papillary adenocarcinoma and 3 SCCs. Additionally, a patient with ADC presented insertion at nucleotide 2300 of 9 nucleotides (Table I). Patients with EGFR mutations were categorized into two groups, those who carried 'classical' activating mutations (del 19 and L858R) that have been reported to be sensitive to gefitinib (25), and those who had 'other variants' of unknown function. Subsequent analyses were performed according to the mutation categorization.
Table II. Correlation of staining scores of SOX2 and MET to pathological features in NSCLCs.

\begin{tabular}{lrccc}
\hline & \multicolumn{4}{c}{ Wilcoxon mean score } \\
\cline { 2 - 5 } Variable & SOX2 & P-value & MET & P-value \\
\hline Gender & & $<0.0001$ & & 0.0006 \\
Male (n=98) & 87.4 & & 66.8 & \\
Female (n=52) & 53.1 & & 91.9 & \\
Histological type & & $<0.0001$ & & $<0.0001$ \\
ADC (n=80) & 55.1 & & 90.1 & \\
SCC (n=57) & 107.8 & & 54.8 & \\
ADSC (n=9) & 63.3 & & 76.2 & \\
LCC (n=4) & 50.6 & & 76.8 & \\
Tumor differentiation & & 0.036 & & 0.113 \\
Well (n=13) & 52.8 & & 93.5 & \\
Moderate and & 77.7 & & 73.8 & \\
poor (n=137) & & & & \\
Tumor size (cm) & & 0.014 & & 0.012 \\
$<5$ (n=106) & 70.2 & & 81.1 & \\
$\geq 5$ (n=44) & 88.3 & & 61.9 & \\
Lymph node & & 0.016 & & \\
metastasis & & & & \\
Positive (n=76) & 67.5 & & 78.7 & \\
Negative (n=74) & 83.7 & & & \\
\hline
\end{tabular}

The non-parametric Kruskal-Wallis test was performed and the Wilcoxon mean score was used to evaluate the correlation of the staining scores of SOX2 and MET expression with pathological features. ADC, adenocarcinoma; SCC, squamous cell carcinoma; ADSC, adenosquamous carcinoma; LCC, large cell carcinoma.

Expression of MET and SOX2 in NSCLC and correlation with EGFR mutation. Results of immunohistochemical studies in lung carcinomas are summarized in Tables II, III and IV. MET positive reactivity of $2+$ and $3+$ was observed in $81.3 \%$ ADCs and $52.6 \%$ of SCCs; there was significant difference between these two entities $(\mathrm{P}=0.001)$. The data indicated that $91.3 \%$ (73/80) ADCs, 82.5\% (47/57) SCCs in addition to all ADSCs and LCCs expressed MET in 1+ to 2+. The expression score of $\leq 1+$ of SOX2 was observed in $81.3 \%$ of ADCs vs. in $21.1 \%$ of SCCs $(\mathrm{P}<0.0001$, Table III, Fig. 2$)$. SOX2 with $2+$ and $3+$ positivity was expressed in $18.8 \%$ of ADCs and $78.9 \%$ of SCCs (Fig. 2). The expression level of SOX2 was higher in SCCs than ADCs $\left(\chi^{2}=49, \mathrm{P}<0.001\right)$.

Non-parametric ANOVA was used to evaluate staining scores of SOX2 and MET. The mean of Wilcoxon Scores were involved in analyzing the staining scores of SOX2 and MET expression with pathological features (Table II). The staining scores of SOX2 were significantly different among patients of different gender, histological types, and tumor dimension. We also found that SOX2 was preferentially highly expressed in males and in SCCs, in contrast to MET that was expressed in females and ADCs (Table II). In addition, NSCLCs with 
Table III. Relationship between different histopathological types with the expression level of MET and SOX2.

\begin{tabular}{|c|c|c|c|c|c|c|c|c|c|c|}
\hline \multirow[b]{2}{*}{ Histopathological types } & \multicolumn{5}{|c|}{ MET staining grade } & \multicolumn{5}{|c|}{ SOX2 staining grade } \\
\hline & 0 & $1+$ & $2+$ & $3+$ & P-value & 0 & $1+$ & $2+$ & $3+$ & P-value \\
\hline $\mathrm{ADC}$ & 3 & 12 & 61 & 4 & 0.024 & 53 & 12 & 15 & 0 & $<0.0001$ \\
\hline ADSC & 0 & 2 & 7 & 0 & & 6 & 1 & 1 & 1 & \\
\hline $\mathrm{LCC}$ & 0 & 1 & 3 & 0 & & 3 & 0 & 1 & 0 & \\
\hline SCC & 10 & 17 & 30 & 0 & & 9 & 3 & 39 & 6 & \\
\hline
\end{tabular}

Table IV. Correlation among NSCLCs in the expression of MET and SOX2 with EGFR mutation.

\begin{tabular}{|c|c|c|c|c|c|c|c|c|c|c|c|c|c|c|c|c|c|c|c|c|}
\hline \multirow{3}{*}{$\begin{array}{l}\text { Histopatho- } \\
\text { logical types }\end{array}$} & \multicolumn{10}{|c|}{ EGFR non-mutation } & \multicolumn{10}{|c|}{ EGFR mutation } \\
\hline & \multicolumn{5}{|c|}{ MET staining grade } & \multicolumn{5}{|c|}{ SOX2 staining grade } & \multicolumn{5}{|c|}{ MET staining grade } & \multicolumn{5}{|c|}{ SOX2 staining grade } \\
\hline & 0 & $1+$ & $2+$ & $3+$ & P-value & 0 & $1+$ & $2+$ & $3+$ & P-value & 0 & $1+$ & $2+$ & $3+$ & P-value & 0 & $1+$ & $2+$ & $3+$ & P-value \\
\hline $\mathrm{ADC}$ & 3 & 11 & 34 & 4 & 0.127 & 33 & 8 & 11 & 0 & $<0.0001$ & 0 & 1 & 27 & 0 & 0.069 & 20 & 4 & 4 & 0 & 0.334 \\
\hline ADSC & 0 & 1 & 7 & 0 & & 6 & 1 & 0 & 1 & & 0 & 1 & 0 & 0 & & 0 & 0 & 1 & 0 & \\
\hline LCC & 0 & 3 & 1 & 0 & & 3 & 0 & 1 & 0 & & 0 & 0 & 0 & 0 & & 0 & 0 & 0 & 0 & \\
\hline $\mathrm{SCC}$ & 10 & 17 & 27 & 0 & & 7 & 3 & 38 & 6 & & 0 & 0 & 3 & 0 & & 2 & 0 & 1 & 0 & \\
\hline
\end{tabular}

EGFR, epidermal growth factor receptor gene.

moderate or poor differentiation, tumor dimension $\geq 5 \mathrm{~cm}$, and lymph node invasion were highly expressed with SOX2. Interestingly, MET activation was otherwise activated in tumors with a dimension $<5 \mathrm{~cm}$ and not associated with lymph node involvement and histological differentiation.

In our study, 13 well-differentiated ADCs showed low expression of SOX2. The strongest staining grade of SOX2 was $2+$ in all ADCs in comparison to $3+$ in SCCs. SOX2 was highly expressed in $78.9 \%$ (45/57) of SCCs, compared to $18.8 \%(15 / 80)$ of ADCs $(\mathrm{P}<0.001)$. High expression of SOX2 appeared in $14.3 \%(4 / 28)$ of ADCs, compared to $33.3 \%(1 / 3)$ of SCCs out of the 32 tumors bearing EGFR mutation. Of the 28 ADCs, the $24(85.7 \%)$ with an EGFR mutation showed low SOX2 expression including all 3 BACs, one mucinous carcinoma and one papillary adenocarcinoma (Table I). SOX2 expression was not associated with tumor differentiation and lymph node invasion in ADCs. A higher expression level of SOX2 was present in NSCLCs without an EGFR mutation $(90.5 \%, \mathrm{n}=118)$ compared to those with an EGFR mutation $(9.5 \%, \mathrm{n}=32)\left(\chi^{2}=9.02, \mathrm{P}=0.0027\right)$. Our data denote that $11.3 \%$ NSCLCs (17/150) had EGFR deletions in exon 19, and $5.9 \%$ (1/17) highly expressed SOX2 in contrast to $46.6 \%(62 / 133)$ of those without this mutation. Likewise, MET expression was demonstrated in $84.9 \%$ non-mutated EGFR tumors and in $15.1 \%$ mutated tumors. When stratified by histological types, $96.4 \%$ (27/28) were found to have a high level of MET expression among ADCs with EGFR mutation vs. 73.1\% (38/52) of those without EGFR mutation $(\mathrm{P}=0.014)$. We also found that ADCs with exon 19 deletion were overexpressed in MET and lowly expressed in SOX2 in contrast to those ADCs without exon 19 deletion $(\mathrm{P}=0.033$ and 0.033 , respectively).
SCCs also had mutation in exons 18 and 21, and a significantly low level of SOX2 expression was detected in the two SCCs with a mutation in exon 21 compared with those without this mutation. A correlation was not found between mutation in exons 18 and 20 and overexpression of MET and SOX2 among both ADCs and SCCs. A strong expression of MET appeared in $81.3 \%$ ADCs $(n=80)$ and $52.6 \%$ SCCs $(n=57)$; a similar expression was observed in $77.8 \%$ ADSCs $(n=9)$ and $75 \%$ LCCs $(n=4)$. Furthermore, SOX2 expression was inversely correlated with the expression of MET in NSCLC and mainly in non-mutated NSCLC $(r=-0.42, \mathrm{P}<0.0001)$. Expression of SOX2 among SCCs was found to be inversely correlated with MET expression ( $\mathrm{r}=-0.40 ; \mathrm{P}=0.002)$, but not in ADCs and ADSCs.

ADSC has parenchyma both of ADC and SCC. One ADSC specimen was observed to have a mutation in EGFR exon 19 (del K745-E749) with low MET expression and overexpression of SOX2. Further observation revealed a tendency for SOX2 to be expressd in the SCC partition of ADSCs, and for MET to be expressed in the part of ADC with a grade of mild to strong staining (Fig. 3). This is an interesting phenomenon inferring that MET and SOX2 may be specific to ADC and SCC, respectively. An ADSC having an EGFR mutation (case 57) showed SOX2 overexpression and low expression of MET. We speculated that the mutation may occur in the part of ADC.

All LCCs showed a low expression of SOX2 and 75\% also showed low MET expression. MET was preferentially expressed in ADCs compared to SCCs. None of the 4 LCCs were found to have an EGFR mutation in our study, and immunostaining of MET presented moderate expression in $75 \%(3 / 4)$ LCCs, but 3 LCCs showed negative SOX2 staining, with a staining score of only 1 (moderate staining). 

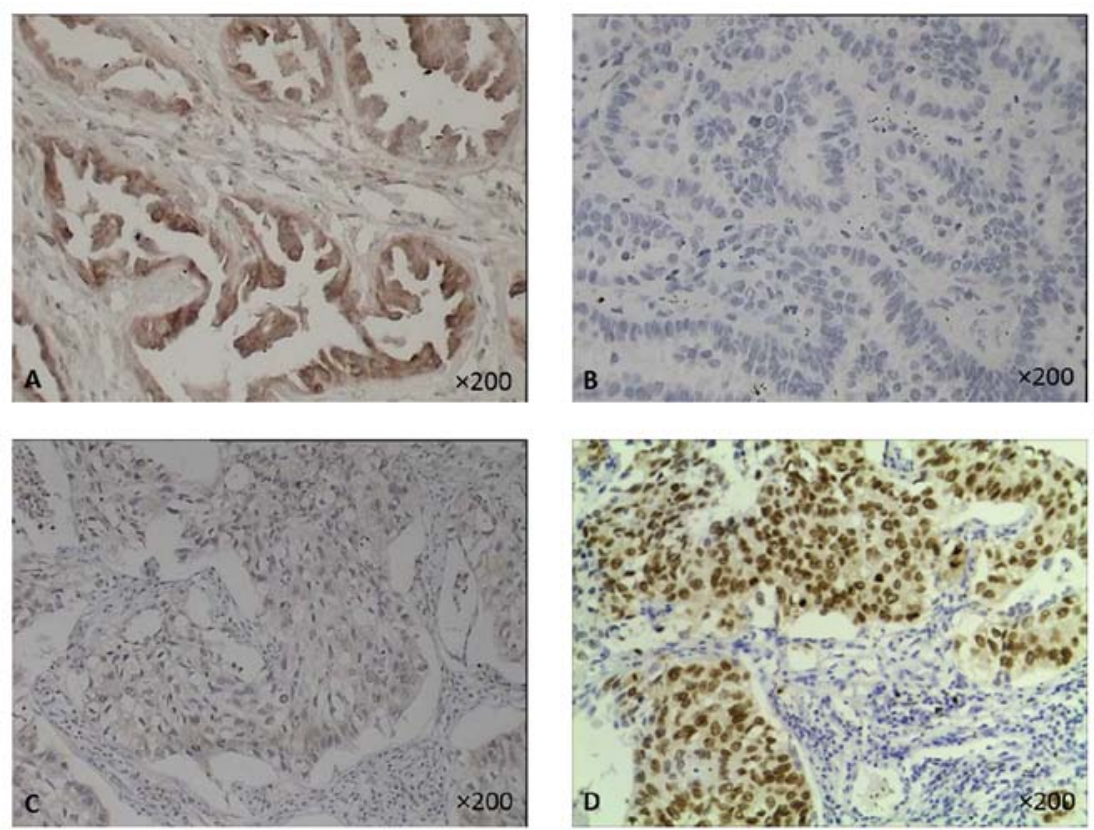

Figure 2. Immunohistochemical staining of lung NSCLC samples. (A and B) Example of ADC tumor. SOX2 and MET antibodies show different staining patterns with MET cytoplasmic staining being positive with a score of 6 , and SOX2 being negative (magnification x200). (C and D) SOX2 and MET antibodies also show positive staining in a tumor of SCC; however with a low MET score of 2 and a high SOX2 score of 9, in opposition to ADC (magnification x200).
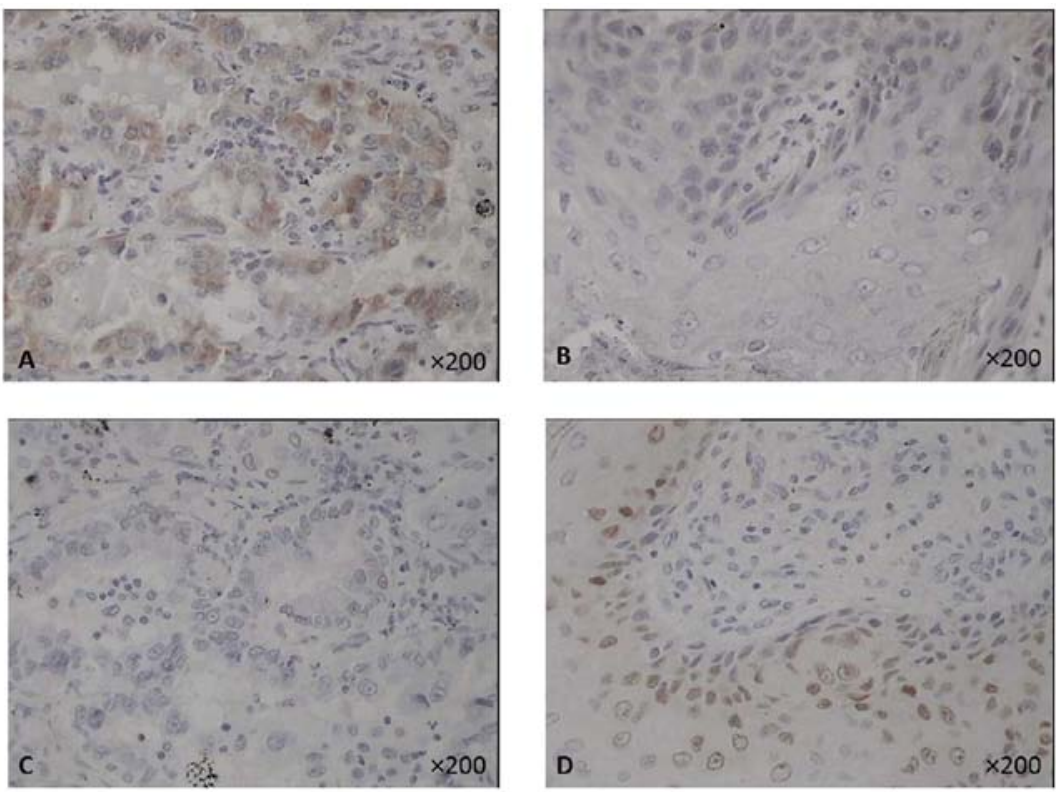

Figure 3. Representative staining for MET and SOX2 in an ADSC. (A and B) MET stain in ADC and SCC partitions of the ADSC sample with a MET staining score of 1 in the ADC part, but negative in the SCC part (magnification x200). (C and D) SOX2 staining in ADC and SCC partitions, respectively, and only stained in the SCC part with a staining score of 1 (magnification $\mathrm{x} 200$ ).

\section{Discussion}

The tyrosine kinase receptor EGFR pathway has been extensively studied in NSCLC since gefitinib and erlotinib have been used in NSCLC, with a clinical response more commonly observed in the ADC/BAC histology, in non-smokers, women and patients of East-Asian ethnicity (5). EGFR mutations likely play a role in the sensitivity to TKIs, with gene mutations primarily found in exons 18, 19, 20 and 21. These exons comprise the first four exons that encode the tyrosine kinase domain. Many different EGFR mutations have been reported in the literature; however, only four mutations have been associated with TKIs sensitivity. These mutations are point mutations in exons 18 (G719A/C) and 21 (L858R and L861Q) and an in-frame deletion in exon 19 from codon 746-750 (E746-A750del) (26). In patients, 90\% of the EGFR mutations associated with gefitinib sensitivity are either del E746-A750 or L858R. Sakai et al reported that in the delE746-A750 mutation, dimerization and phosphorylation of EGFR were observed in the absence of ligand stimulation, leading to constitutive 
phosphorylation of EGFR and its downstream targets (27). Other studies found that the catalytic efficiency $\left(\mathrm{k}_{\mathrm{cat}} / \mathrm{K}_{\mathrm{M}}\right)$ of the L858R mutant is $\sim 20$-fold higher than that of the wild-type kinase domain, suggesting that the wild-type kinase domain is inhibited and the L858R mutant is constitutively active (28). SOX2 is a key transcription factor, which is decisively involved in stabilizing embryonic stem cells in a pluripotent state (29). Advances of stem cell biology in the last years accumulated evidence that not only embryonic but also cancer stem cells exist. Properties of such cancer stem cells include self-renewal which drives tumorigenesis, and aberrant differentiation that contributes to cellular heterogeneity. It was suggested that tumors contain a cellular population that retains key stem cell properties (30), which have gene expression signatures closely related to embryonic stem cells (31). High SOX2 expression was found in breast cancer (32), testicular germ cell tumors (33) and gastric adenocarcinoma (21). Expression of SOX2 protein has not been extensively studied in lung cancer; however, a recent study demonstrates that SOX2 is strongly and diffusely expressed in about $90 \%$ of pulmonary SCC and $20 \%$ of ADC (34). In the current study, all well-differentiated ADCs and $85.7 \%$ ADCs with an EGFR mutation expressing low levels of SOX 2 suggest that SOX2 expression is associated with differentiation of ADC and inversely correlated with the activation of EGFR.

Recent findings have shown that MET amplification is associated with gefitinib resistance during therapy (8). The MET gene is composed of 21 exons and 20 introns $(35,36)$. The function of MET in human tumors can be enhanced by mutation or amplification, leading to oncogenic changes including cell proliferation, reduced apoptosis, angiogenesis and altered cytoskeletal function. MET and its ligand HGF have been reported to be mis- and overexpressed in head and neck squamous cell carcinoma (HNSCC); this situation may lead to the constitutive activation of the receptor tyrosine kinase system. Using high-throughput analyses in both cell lines and in patients with lung cancer it has been shown that subpopulations of cells with MET amplification exist prior to drug exposure (37). In the present study, we found that the EGFR mutation was not accompanied with expression of the SOX2 and MET genes. When comparing the expression of SOX 2 to exon 19 deletion, we found that SOX 2 overexpression was present in $46.6 \%$ non-mutated and $5.9 \%$ mutated NSCLCs tumors, respectively; a similar difference was demonstrated in the overexpression of MET with $84.9 \%$ vs. $15.1 \%$, respectively. ADCs with the exon 19 deletion of the EGFR gene also have a high level of MET expression and a low level of SOX2 suggesting that ADCs may have a different molecular pathway to maintain its progression compared to SCCs. The data demonstrate that SOX2 activation is common and necessary for SCC.

In this study, all cases were selected before target-drug exposure. We found that MET overexpression was prominent in ADCs (81.3\%) in conjunction to SCCs (52.6\%) despite EGFR mutation. MET overexpression appeared most frequently in adenocarcinomas. MET was also found to be deleted in ADC/ BACs and SCCs (5); and we also demonstrated that low expression in both MET and SOX2 presented in BACs, mucinous adenocarcinoma and papillary adenocarcinoma with an EGFR mutation. This low expression of MET may result from MET deletion and suggest that the overexpression of MET is mainly harbored in tubular ADCs.

The current study disclosed that the MET activation status was associated with gender, histology and tumor size and the incidence of MET expression level was higher in tubular ADC than in SCC. Former studies showed paradoxical results in MET amplification with the clinicopathological features among NSCLC. Okuda et al reported that increased MET gene copy number (GCN) was observed in $5.6 \%$ of patients with NSCLC, who were men and smokers, whereas no difference in MET GCN status was seen in terms of histological types (38). In contrast, other studies reported that MET amplification (or high GCN) was not significantly associated with gender, smoking history or histology $(39,40)$. However, Go et al reported that most patients with true MET amplification had SCC (41). Our data demonstrate that MET expression is not only associated with females, ADC and tumor size, but is more prevalent in ADCs with exon 19 deletion. This change is prior to the exposure to targeted therapy of TKI. Our result is also consistent with a previous report in a cell line (42). Our results suggest that MET expression may be more involved in the tumorigenesis of exon 19-activated ADCs and a combination strategy could benefit ADC patients showing MET overexpression and exon 19 deletion.

We have demonstrated that SOX2 expression was higher among male patients, and in SCCs with moderate or poor differentiation, larger tumor dimension and lymph node invasion. SOX2 overexpression was more prevalent in SCCs than in ADCs. SOX2 is reported to be a master pluripotency controller and has recently been identified as a novel major oncogene, recurrently amplified and activated in SCC $(43,44)$. These studies have used a similar strategy of chromosomal aberrations screening to identify the SOX2 locus as one of the most frequently amplified sites over the SCC genome. They have further highlighted the recurrent SOX2 activation and its necessary role for squamous cell survival. Finally, they have shown that SOX2 is also involved in the early steps of lung SCC, and participates to transform human bronchial epithelial cells. Furthermore, SOX 2 overexpression can induce the expression of the squamous markers p63 and keratin 6, supporting the idea that SOX2 might be implicated in SCC differentiation (44). We also found that SOX2 was expressed at a higher level in moderately or poorly differentiated SCCs and the portion of SCC in ADSCs. However, neither study assessed the impact of the recurrent activation of SOX2 in advanced primary tumors nor how SOX2 may mechanistically participate in tumor progression and aggressiveness. The present study adds to the current state of knowledge and suggests novel perspectives on the multiples roles that SOX2 exerts in SCC carcinogenesis. In our study population, we found that $78.9 \%$ of SCCs strongly expressed SOX2 presenting a higher proportion of SOX2 overexpression than ADCs. The data indicate a discrepancy in the characteristics of SCC and ADC. Current comparative genomic hybridization studies have demonstrated that $>90 \%$ of SCCs and $20 \%$ of ADCs have copy number gain involving 3q26,4, essentially the same proportions that have been demonstrated to have high level SOX2 expression at the protein level (34). Of note, these studies have found high level amplification only in SCC (45). These observations warrant additional studies to determine the molecular mechanisms of 
SOX2 expression in ADC. Because this pilot study was biased to include surgically resectable, moderately and poorly differentiated SCCs, there were too few well-differentiated tumors to perform a well-powered statistical analysis. However, we hypothesize that SOX2 may play an important role in the carcinogenesis of SCCs. Former studies suggest that overexpression of SOX2 may be an important way to activate the Nanog/Oct4/SOX2 pathway, which promotes tumor cell proliferation and is associated with short survival time of patients in stage I ADC (46). SOX2 is also activated in more advanced SCC tumors as others have reported $(23,34)$. We conclude that SOX 2 is activated not only by overexpression, but also through amplification as also mentioned by others $(23,47)$. SOX2 gene overexpression is more common in SCCs with poor differentiation and lymph node metastasis, as well as in larger tumors. The incidence of SOX2 overexpression is associated with SCC growth and progression. We also speculate that the SOX2 gene is not only activated by amplification but may be regulated by other factors to promote its transcription and which in turn may affect its downstream genes.

ADSCs are morphologically mixed tumors that contain the two cell components ADC and SCC. To understand if they are a 'simple' mix of ADC and SCC or if they present distinct molecular specificities, as compared with the molecular characterization of both components, Bastide et al found that genes were differentially expressed when comparing ADCs with SCCs, ADSCs with SCCs and ADCs with ADSCs, respectively (48). Through our immunohistochemical assay, we observed, when comparing the three histological subtypes that SOX2 was expressed mainly in SCCs and the part of SCC in ADSCs and negatively or weakly expressed in ADC and the ADC part of ADSC, respectively. Furthermore, according to our results, an ADSC sample also harbored the EGFR mutation, but the others did not have an EGFR mutation. Collectively the data indicate that ADSCs were classified as an intermediate lesion between the ADCs and SCCs, some being similar to ADCs, others to SCCs. ADSCs could be considered as a mix of ADCs and SCCs with varied proportions, but more complex than simple mixtures of ADC and SCC components. SOX2 could be regarded as a specific marker in SCC. We also demonstrated that SOX2 was preferentially expressed in ADSCs compared to the MET gene (Figs. 2 and 3). Future studies with an increased sample size would assist in the illustration of the molecular specificity of ADSCs.

\section{Acknowledgements}

We appreciate Xue-Jing Chen, Li Zhang, Ying-Li Zhao, Chong-Li Wang and Chen Zhang for their support in sample collection. The study was supported by the Beijing Natural Science Foundation for the Youth grant (QN2009-035), by the Beijing Foundation for Distinguished Scientists grant 2009D003013000001 and by awards from the Beijing Board of Health.

\section{References}

1. Travis WD, Brambilla E, Muller-Hermelink HK and Harris CC (eds): World Health Organization Classification of Tumours, Pathology and Genetics: Tumours of the Lung, Pleura, Thymus and Heart. IARC Press, Lyon, 2004.
2. Laird AD and Cherrington JM: Small molecule tyrosine kinase inhibitors: clinical development of anticancer agents. Expert Opin Investig Drugs 12: 51-64, 2003.

3. Maulik G, Kijima T and Salgia R: Role of receptor tyrosine kinases in lung cancer. Methods Mol Med 74: 113-125, 2003.

4. Lamorte $\mathrm{L}$ and Park M: The receptor tyrosine kinases: role in cancer progression. Surg Oncol Clin N Am 10: 271-288, viii, 2001.

5. Fukuoka M, Yano S, Giaccone G, et al: Multi-institutional randomized phase II trial of gefitinib for previously treated patients with advanced non-small cell lung cancer (The IDEAL 1 Trial) [corrected]. J Clin Oncol 21: 2237-2246, 2003.

6. Kris MG, Natale RB, Herbst RS, et al: Efficacy of gefitinib, an inhibitor of the epidermal growth factor receptor tyrosine kinase, in symptomatic patients with non-small cell lung cancer: a randomized trial. JAMA 290: 2149-2158, 2003.

7. Shepherd FA, Rodrigues Pereira J, Ciuleanu T, et al: Erlotinib in previously treated non-small cell lung cancer. N Engl J Med 353: 123-132, 2005.

8. Engelman JA, Zejnullahu K, Mitsudomi T, et al: MET amplification leads to gefitinib resistance in lung cancer by activating ERBB3 signaling. Science 316: 1039-1043, 2007.

9. Naldini L, Weidner KM, Vigna E, et al: Scatter factor and hepatocyte growth factor are indistinguishable ligands for the MET receptor. EMBO J 10: 2867-2878, 1991.

10. Bottaro DP, Rubin JS, Faletto DL, et al: Identification of the hepatocyte growth factor receptor as the c-met proto-oncogene product. Science 251: 802-804, 1991.

11. Birchmeier C, Birchmeier W, Gherardi E and van de Woude GF: Met, metastasis, motility and more. Nat Rev Mol Cell Biol 4: 915-925, 2003.

12. Maulik G, Shrikhande A, Kijima T, Ma PC, Morrison PT and Salgia R: Role of the hepatocyte growth factor receptor, c-Met, in oncogenesis and potential for therapeutic inhibition. Cytokine Growth Factor Rev 13: 41-59, 2002.

13. Cooper CS, Park M, Blair DG, et al: Molecular cloning of a new transforming gene from a chemically transformed human cell line. Nature 311: 29-33, 1984.

14. Chen JT, Lin TS, Chow KC, et al: Cigarette smoking induces overexpression of hepatocyte growth factor in type II pneumocytes and lung cancer cells. Am J Respir Cell Mol Biol 34: 264-273, 2006.

15. Nakamura Y, Niki T, Goto A, et al: c-Met activation in lung adenocarcinoma tissues: an immunohistochemical analysis. Cancer Sci 98: 1006-1013, 2007.

16. Tsao MS, Liu N, Chen JR, et al: Differential expression of Met/ hepatocyte growth factor receptor in subtypes of non-small cell lung cancers. Lung Cancer 20: 1-16, 1998.

17. Beau-Faller M, Gaub MP, Schneider A, et al: Allelic imbalance at loci containing FGFR, FGF, c-Met and HGF candidate genes in non-small cell lung cancer sub-types, implication for progression. Eur J Cancer 39: 2538-2547, 2003.

18. Que J, Luo X, Schwartz RJ and Hogan BL: Multiple roles for Sox 2 in the developing and adult mouse trachea. Development 136: 1899-1907, 2009.

19. Gontan C, De Munck A, Vermeij M, Grosveld F, Tibboel D and Rottier R: Sox2 is important for two crucial processes in lung development: branching morphogenesis and epithelial cell differentiation. Dev Biol 317: 296-309, 2008.

20. Park KS, Wells JM, Zorn AM, et al: Transdifferentiation of ciliated cells during repair of the respiratory epithelium. Am J Respir Cell Mol Biol 34: 151-157, 2006.

21. Tsukamoto T, Mizoshita T, Mihara M, et al: Sox 2 expression in human stomach adenocarcinomas with gastric and gastric-andintestinal-mixed phenotypes. Histopathology 46: 649-658, 2005.

22. Li XL, Eishi Y, Bai YQ, et al: Expression of the SRY-related HMG box protein SOX2 in human gastric carcinoma. Int J Oncol 24: 257-263, 2004.

23. Hussenet T, Dali S, Exinger J, et al: SOX2 is an oncogene activated by recurrent $3 q 26.3$ amplifications in human lung squamous cell carcinomas. PLoS One 5: e8960, 2010.

24. Ebright MI, Zakowski MF, Martin J, et al: Clinical pattern and pathologic stage but not histologic features predict outcome for bronchioloalveolar carcinoma. Ann Thorac Surg 74: 1640-1647, 2002.

25. Lynch TJ, Bell DW, Sordella R, et al: Activating mutations in the epidermal growth factor receptor underlying responsiveness of non-small cell lung cancer to gefitinib. N Engl J Med 350: 2129-2139, 2004.

26. Riely GJ, Politi KA, Miller VA and Pao W: Update on epidermal growth factor receptor mutations in non-small cell lung cancer. Clin Cancer Res 12: 7232-7241, 2006. 
27. Sakai K, Arao T, Shimoyama T, et al: Dimerization and the signal transduction pathway of a small in-frame deletion in the epidermal growth factor receptor. FASEB J 20: 311-313, 2006.

28. Zhang X, Gureasko J, Shen K, Cole PA and Kuriyan J: An allosteric mechanism for activation of the kinase domain of epidermal growth factor receptor. Cell 125: 1137-1149, 2006.

29. Masui S, Nakatake Y, Toyooka Y, et al: Pluripotency governed by Sox 2 via regulation of Oct3/4 expression in mouse embryonic stem cells. Nat Cell Biol 9: 625-635, 2007.

30. Wicha MS, Liu S and Dontu G: Cancer stem cells: an old idea - a paradigm shift. Cancer Res 66: 1883-1895, 2006.

31. Ben-Porath I, Thomson MW, Carey VJ, et al: An embryonic stem cell-like gene expression signature in poorly differentiated aggressive human tumors. Nat Genet 40: 499-507, 2008.

32. Chen Y, Shi L, Zhang L, et al: The molecular mechanism governing the oncogenic potential of SOX2 in breast cancer. J Biol Chem 283: 17969-17978, 2008.

33. Biermann K, Heukamp LC, Steger K, et al: Genome-wide expression profiling reveals new insights into pathogenesis and progression of testicular germ cell tumors. Cancer Genomics Proteomics 4: 359-367, 2007.

34. Sholl LM, Long KB and Hornick JL: Sox 2 expression in pulmonary non-small cell and neuroendocrine carcinomas. Appl Immunohistochem Mol Morphol 18: 55-61, 2010.

35. Maestrini E, Tamagnone L, Longati P, et al: A family of transmembrane proteins with homology to the MET-hepatocyte growth factor receptor. Proc Natl Acad Sci USA 93: 674-678, 1996.

36. Comoglio PM and Boccaccio C: The HGF receptor family: unconventional signal transducers for invasive cell growth. Genes Cells 1: 347-354, 1996.

37. Turke AB, Zejnullahu K, Wu YL, et al: Preexistence and clonal selection of MET amplification in EGFR mutant NSCLC. Cancer Cell 17: 77-88, 2010

38. Okuda K, Sasaki H, Yukiue H, Yano M and Fujii Y: Met gene copy number predicts the prognosis for completely resected non-small cell lung cancer. Cancer Sci 99: 2280-2285, 2008.

39. Bean J, Brennan C, Shih JY, et al: MET amplification occurs with or without T790M mutations in EGFR mutant lung tumors with acquired resistance to gefitinib or erlotinib. Proc Natl Acad Sci USA 104: 20932-20937, 2007.
40. Cappuzzo F, Marchetti A, Skokan M, et al: Increased MET gene copy number negatively affects survival of surgically resected non-small cell lung cancer patients. J Clin Oncol 27: 1667-1674, 2009.

41. Go H, Jeon YK, Park HJ, Sung SW, Seo JW and Chung DH: High MET gene copy number leads to shorter survival in patients with non-small cell lung cancer. J Thorac Oncol 5: 305-313, 2010.

42. McDermott U, Pusapati RV, Christensen JG, Gray NS and Settleman J: Acquired resistance of non-small cell lung cancer cells to MET kinase inhibition is mediated by a switch to epidermal growth factor receptor dependency. Cancer Res 70: 1625-1634, 2010

43. Hussenet T and Du Manoir S: SOX2 in squamous cell carcinoma: Amplifying a pleiotropic oncogene along carcinogenesis. Cell Cycle 9: 1480-1486, 2010

44. Bass AJ, Watanabe $\mathrm{H}$, Mermel $\mathrm{CH}$, et al: SOX2 is an amplified lineage-survival oncogene in lung and esophageal squamous cell carcinomas. Nat Genet 41: 1238-1242, 2009.

45. Bjorkqvist AM, Husgafvel-Pursiainen K, Anttila S, et al: DNA gains in $3 q$ occur frequently in squamous cell carcinoma of the lung, but not in adenocarcinoma. Genes Chromosomes Cancer 22: 79-82, 1998.

46. Sholl LM, Barletta JA, Yeap BY, Chirieac LR and Hornick JL: Sox 2 protein expression is an independent poor prognostic indicator in stage I lung adenocarcinoma. Am J Surg Pathol 34: 1193-1198, 2010.

47. McCaughan F, Pole JC, Bankier AT, et al: Progressive 3q amplification consistently targets SOX2 in preinvasive squamous lung cancer. Am J Respir Crit Care Med 182: 83-91, 2010.

48. Bastide K, Ugolin N, Levalois C, Bernaudin JF and Chevillard S: Are adenosquamous lung carcinomas a simple mix of adenocarcinomas and squamous cell carcinomas, or more complex at the molecular level? Lung Cancer 68: 1-9, 2010. 